\title{
然
}

\section{LOS GRUPOS DE INTERÉS Y SU IMPORTANCIA EN LA PROPUESTA DE VALOR DE LAS EMPRESAS}

Los grupos de interés son un elemento fundamental de la relación de las empresas con su entorno de actividad y están estrechamente vinculados con su capacidad para conseguir sus objetivos económicos y sobrevivir en el largo plazo. Representan a dicho entorno, social y medioambiental, experimentando el impacto de la actividad de las empresas y canalizando sus expectativas y demandas económicas, pero también sociales y medioambientales, hacia las mismas. Este triple nivel de exigencia los relaciona con el concepto de responsabilidad social corporativa, de gran actualidad. El objetivo del artículo es comprender la naturaleza de los grupos de interés y su influencia en las empresas para entender la necesidad de las empresas de identificarlos en su entorno y diseñar una propuesta de valor que abarque tanto aspectos económicos como sociales y medioambientales.

Palabras clave: stakeholders, responsabilidad social corporativa, RSC, sostenibilidad corporativa, legitimidad.

Clasificación JEL: D21, D63, M11, M14, M33.

\section{Introducción}

La necesidad de las empresas de relacionarse con su entorno, en materia de intercambios, les obliga a diseñar cuidadosamente su propuesta de valor (Osterwalder y Pigneur, 2010). Para conseguirlo, una tarea básica es identificar las personas y colectivos con los que se ven obligadas a interactuar, más allá de clientes y proveedores, y comprender sus demandas o necesidades. Así la empresa evita «desconectar» de la realidad en que opera y

\footnotetext{
*Departamento de Organización de Empresas. Universidad Complutense de Madrid.

Versión de noviembre de 2017.
}

consigue ser aceptada obteniendo legitimidad y colaboración de dichos colectivos para actuar y sobrevivir en el largo plazo (Wood, 2010).

Las relaciones de la empresa se instrumentalizan a través de los llamados grupos de interés, por ello resulta necesario comprender el concepto y su importancia para la organización. Debido al impacto de la actuación sobre el entorno, a la empresa se le exige un aporte de valor que va más allá del puramente económico, abarcando cuestiones de tipo social y medioambiental. Estos tres aspectos están relacionados con lo que se considera responsabilidad social corporativa (RSC), una materia de gran actualidad e interés en las $D$ 
últimas décadas (Waddock et al., 2002; Aguinis y Glavas, 2012). Son los grupos de interés los que evalúan la propuesta de valor de la empresa, ya que conceptualmente, por un lado, resultan afectados por su actividad y/o poseen capacidad de influencia en la misma (Freeman, 1984) y, por otro lado, actúan en representación de la sociedad y el medio natural. Estos colectivos han ido aumentando las expectativas respecto a que las corporaciones se comporten de manera más responsable (Auld et al., 2008), tanto en el plano económico como en el social y medioambiental, especialmente a la luz de los escándalos que pueden surgir sobre el oportunismo y la actuación irresponsable de las empresas.

El objetivo de este artículo es revisar y aclarar el concepto de grupo de interés, analizando su importancia para toda empresa que pretenda mantener su actividad en el largo plazo, así como entender la relación entre los conceptos de grupo de interés y RSC. El artículo se estructura en tres bloques, comenzado con un repaso del concepto de grupos de interés, seguido de una relación de criterios básicos para su clasificación. Por último, se analiza la influencia de los grupos de interés en las empresas, su estrategia y su modo de actuación. El documento se basa en el contenido de la tesis doctoral de González Masip (2017).

\section{El concepto de grupo de interés}

Según la perspectiva de los grupos de interés, la empresa ha dejado de considerarse simplemente una caja negra a la que ciertos proveedores facilitan recursos que permiten, previa transformación, satisfacer de productos y servicios a clientes (flujo de entrada-salida), gracias a la participación de los empleados en los procesos de creación de valor, y a la vigilancia y control de propietarios y accionistas. La perspectiva de grupos de interés considera que la empresa es una entidad que interactúa de manera bidireccional con una gran variedad de individuos y colectivos, llamados grupos de interés o stakeholders. Entre estos también se incluirían comunidades, Gobiernos, grupos políticos, medios de comunicación y otros (Freeman, 1984).

Los grupos de interés permiten responder a la pregunta « ¿ante quién es responsable la empresa?» (Maignan et al., 1999: 457) e influyen de manera decisiva en la manera en que las empresas implementan su misión y objetivos, así como sus estrategias de responsabilidad social y medioambiental. En general, el concepto de grupo de interés facilita la comprensión de la naturaleza de la RSC por ser parte del contexto en que las empresas operan y condicionan la actuación social de éstas. Sin embargo, independientemente de su posicionamiento social y medioambiental, toda empresa debe considerar los grupos de interés existentes en sus mercados y entornos de actuación, ya que entre estos también se encuentran clientes, proveedores, inversores y empleados, los cuales son actores clave en sus modelos de negocio (Osterwalder y Pigneur, 2010). Todos ellos son fundamentales e influyentes en los resultados económicos de la empresa, y presentan determinadas exigencias, de naturaleza ética o no. En la Tabla 1 se muestra una serie de definiciones y aproximaciones al concepto de grupo de interés.

Los grupos de interés, que se relacionan activa o pasivamente con las empresas, pueden ser tanto colectivos de personas, formales o informales, como individuos, con cierto perfil, actuando a título particular. Estos $\triangleright$ 
TABLA 1

DEFINICIONES DEL CONCEPTO GRUPOS DE INTERÉS

\begin{tabular}{|c|c|}
\hline Autores & Definición de grupos de interés o stakeholders \\
\hline $\begin{array}{l}\text { Stanford Research Institute } \\
\text { (1963), citado en Freeman y Reed } \\
(1983: 89)\end{array}$ & «Aquellos grupos sin cuyo apoyo la organización podría dejar de existir». \\
\hline Rhenman (1968: 25) & $\begin{array}{l}\text { El término grupo de interés «designa a los individuos o grupos que dependen de la empresa } \\
\text { para la consecución de sus objetivos personales y de los cuales la empresa depende». }\end{array}$ \\
\hline Freeman y Reed (1983: 91) & $\begin{array}{l}\text { «Cualquier grupo o individuo identificable que puede afectar a la consecución de los objetivos } \\
\text { de una organización, o es afectado por la consecución de los objetivos por parte de una } \\
\text { organización». } \\
\text { (Definición amplia: incluye grupos afines y hostiles). }\end{array}$ \\
\hline Freeman y Reed (1983: 91) & $\begin{array}{l}\text { «Cualquier grupo o individuo identificables del cual la organización depende para su continua } \\
\text { supervivencia». } \\
\text { (Definición ajustada: grupos en relación directa con la organización). }\end{array}$ \\
\hline Freeman (1984: 46) & $\begin{array}{l}\text { «Cualquier grupo o individuo que puede afectar o es afectado por la consecución de los } \\
\text { objetivos por parte de una empresa». }\end{array}$ \\
\hline $\begin{array}{l}\text { Clarkson (1994:5), } \\
\text { en Mitchell et al. (1997: 858) }\end{array}$ & $\begin{array}{l}\text { «Grupos o personas que] tienen algún tipo de riesgo como resultado de haber invertido } \\
\text { algún tipo de capital, humano o financiero, algo de valor, en una empresa o se ponen en } \\
\text { riesgo como resultado de las actividades de la empresa». }\end{array}$ \\
\hline Clarkson (1995: 106) & $\begin{array}{l}\text { «Personas o grupos que tienen, o reclaman, propiedad, derechos o intereses en una } \\
\text { corporación o sus actividades, pasado, presente o futuro. Esos derechos o intereses } \\
\text { reclamados son el resultado de transacciones con la empresa, o actuaciones de ésta, y } \\
\text { pueden ser legales o morales, individuales o colectivas». }\end{array}$ \\
\hline Donaldson y Preston (1995: 67) & $\begin{array}{l}\text { «Personas o grupos con intereses legítimos en aspectos procedimentales o sustanciales de } \\
\text { la actividad de la empresa. [...] se identifican por su interés en la corporación, sin importar } \\
\text { si la empresa a cambio presenta algún interés funcional en ellos. La importancia de estos } \\
\text { grupos de interés es un valor intrínseco. Es decir, cada grupo merece consideración en sí } \\
\text { mismo y no simplemente por su capacidad para promover los intereses de algún otro grupo, } \\
\text { como los accionistas». }\end{array}$ \\
\hline Phillips (1999: 32) & «[Grupos o personas] que tienen algo en riesgo en la empresa». \\
\hline Garriga y Melé (2004: 59) & «Personas que afectan o son afectadas por las políticas y prácticas de las empresas». \\
\hline Garriga y Melé (2004: 60) & $\begin{array}{l}\text { [Interpretando a Freeman (1984)] «Aquellos grupos que tienen un interés en o una demanda } \\
\text { hacia la empresa (proveedores, clientes, empleados, accionistas y la comunidad local)». }\end{array}$ \\
\hline
\end{tabular}

individuos se juntan de acuerdo a similitudes en sus intereses, reclamaciones, o derechos (Clarkson, 1995). Con relación al alcance del concepto, las definiciones denominadas ajustadas (o estrechas) harían referencia a grupos concretos y con relación muy directa con la empresa, en aspectos económicos del núcleo de negocio, o un interés moral muy determinado. Esta relación directa implicaría cierta legitimidad en las exigencias de los grupos de interés basada en contratos, intercambios, legalidad, derechos o moralidad. Por otra parte, las definiciones amplias de grupo de interés se aplican prácticamente a cualquier agente social existente en el entorno de la empresa (Mitchell et al., 1997).

Los grupos de interés y su concepto se pueden analizar principalmente desde tres enfoques: (i) normativo, atendiendo a la naturaleza de la demanda de los grupos de interés y cómo los directivos deberían dar respuesta a ésta de acuerdo con la organización y sus principios éticos; (ii) descriptivo, analizando aspectos empíricos (observación) de la existencia de grupos de interés y su relación con la empresa, explicando características, comportamientos, actuaciones y roles específicos desarrollados en la actualidad; y (iii) instrumental, $D$ 
considerando el efecto de la gestión por parte de la empresa de los grupos de interés en la búsqueda de su propio beneficio, es decir, en la consecución de los objetivos de la empresa y la maximización del beneficio en el largo plazo (Donaldson y Preston, 1995; Friedman y Miles, 2006). Típicamente, el enfoque normativo está vinculado a la ética y el instrumental a lo empírico y utilitario (Jones y Wicks, 1999). La gestión o interacción con grupos de interés no es una cuestión exclusiva de empresas socialmente responsables, ni de las actividades sociales en las empresas, y no asegura un tratamiento responsable a los grupos de interés. Se debe separar a nivel de conceptos la gestión de los grupos de interés y el tratamiento responsable de los mismos, ya que se trata de dos cuestiones diferentes (Greenwood, 2007). El hecho de que las relaciones con los grupos de interés sean bidireccionales (grupos que afectan o son afectados por la organización) representa el efecto de la organización en su entorno, incluido el social y medioambiental, y establece un vínculo entre los conceptos de grupo de interés y RSC, que abarca el planteamiento y la actuación de la empresa respecto a su impacto en personas y medioambiente (Watts y Holme, 2000; Van Marrewijk, 2003; Hansen et al., 2011).

\section{Clasificación de grupos de interés}

Rhenman (1968) enumera los grupos de interés como empleados, propietarios, clientes, proveedores, prestamistas y otros muchos grupos. Freeman y Reed (1983), dentro del concepto ajustado de grupo de interés, incluyen a empleados, segmentos de clientes, proveedores, agencias de gobierno, accionistas, determinadas instituciones financieras y otros; y en enfoque amplio incluyen a grupos de protesta, agencias gubernamentales, asociaciones de comercio, competidores, sindicatos, empleados, segmentos de clientes, accionistas, grupos de interés público y otros. Para Clarkson (1995), grupos de interés son empleados, accionistas, directivos, clientes, comunidades, medios de comunicación y nichos de consumidores no clientes. Numerosos autores consideran en sus investigaciones dentro del concepto de grupos de interés a accionistas (David et al., 2007), consumidores (Sen y Bhattacharya, 2001; Christmann y Taylor, 2006), empleados (Friedman, 2009), directivos (Weaver et al., 1999), socios de negocio (Neville et al., 2005), medios de comunicación (Davidson y Worrell, 1988), comunidades locales (Marquis et al., 2007), y otros tipos de grupos (Greening y Gray, 1994).

Cada grupo de interés presenta una serie de particularidades propias, acentuadas por las circunstancias específicas del contexto de actuación de cada empresa. Por ejemplo, los directivos, como grupo, son los únicos con relaciones, de carácter contractual explícito o implícito, con el resto de individuos y grupos de interés en la organización (Mitchell et al., 1997). Otro ejemplo de grupo con características muy específicas es el medioambiente. Mientras que, para algunos autores, el medioambiente estaría representado en sus intereses por comunidades y grupos ecologistas, otros autores consideran que el medio natural es un grupo de interés propio (Hopkins, 2003), calificado específicamente como grupo de interés no-humano (Starik, 1995).

Según Freeman (1984), los grupos de interés se pueden clasificar en internos y externos de acuerdo a los planteamientos de la Teoría de la Organización (Management Theory). Considera grupos de interés internos aquellos que generan cambios en la empresa, como obligar a redefinir objetivos y políticas, y con $\triangleright$ 
los que se pueden establecer relaciones relativamente cómodas y fácilmente gestionables. Los grupos internos serían propietarios y accionistas, clientes, empleados (y directivos) y proveedores. El autor propone que los grupos de interés pertenecientes al llamado entorno, según la Teoría de la Organización, influyen también en la empresa generando cambios, de manera menos controlable y con mayor incertidumbre1. Los grupos considerados externos serían Gobiernos, competidores, asociaciones de consumidores, grupos ecologistas, medios de comunicación y otros grupos de especial interé $^{2}$. Esta situación empuja al autor a plantear otra teoría que permita explicar nuevamente el origen externo de los cambios en la organización, a partir de lo cual propone la teoría de los grupos de interés (Stakeholder Theory).

Clarkson (1995), basándose en el trabajo de Freeman (1984), desarrolla otro criterio de clasificación muy utilizado: los grupos de interés pueden ser primarios o secundarios. Los primeros son aquellos que, debido a la participación continuada en la empresa y la alta interdependencia, son necesarios para la supervivencia de la misma (inversores, empleados, clientes, proveedores, así como Gobiernos y comunidades, responsables ambos de regulaciones, leyes e impuestos que cumplir). Secundarios son aquellos que afectan o influyen, o son afectados o están influidos, por la empresa, pero que, al no estar involucrados en

\footnotetext{
En la actualidad, para diferenciar entre grupos de interés internos y externos, podría resultar más apropiado centrarse en el aspecto de predictibilidad de la influencia de los grupos en la organización, caracterizado por el grado de vinculación con la empresa, la fortaleza de dicha vinculación, así como las barreras al cambio. Según este criterio, por ejemplo, clientes, especialmente los no fidelizados, y trabajadores candidatos podrían considerarse grupos de interés externos al no tener una vinculación contractual con la empresa. Sin embargo permanecen vinculados al estar expuestos al efecto de variables externas a la organización, como la reputación de la empresa (Hansen et al., 2011; Aguinis y Glavas, 2012).

2 Consúltese Freeman (1984) para obtener más información sobre los llamados grupos de especial interés.
}

las transacciones de la empresa, no son vitales para la supervivencia de la misma, pero pueden afectar a su normal funcionamiento (medios de comunicación, grupos políticos u otros grupos capaces de movilizar la opinión pública). Fuera del ámbito académico, el Sociaal Economische Raad, de Países Bajos, clasifica los grupos de interés (SER, 2000) en individuos (accionistas, empleados, consumidores, público) u organismos que los representan (asociaciones de comercio, ONG, organismos de la Administración Pública, sindicatos, etcétera). Las clasificaciones de los grupos de interés se pueden realizar de acuerdo a diferentes criterios o dimensiones, sin que sean estas excluyentes entre sí. En la Tabla 2 se muestra una relación de criterios básicos de clasificación de grupos de interés propuestos por diferentes autores.

Los individuos pueden pertenecer a uno o varios grupos de interés a la vez y adquirir características de todos ellos. Por ejemplo, podrían relacionarse con la empresa como consumidores o ciudadanos de manera externa, y como propietarios, directivos, empleados, o voluntarios de manera interna (Starik y Rands, 1995). La empresa podría optar también por realizar subclasificaciones dentro de los propios grupos de interés de acuerdo a diversos criterios que podrían hacer recomendable una gestión diferenciada de los subgrupos. Un criterio puede ser la presencia en mayor o menor medida en cada grupo de las variables, definidas por Mitchell et al. (1997), poder, legitimidad y urgencia (por ejemplo, aquellos trabajadores que ocupen posiciones más críticas para los resultados empresariales podrían mostrar un mayor poder). Otros criterios podrían tener un enfoque práctico, diferenciando entre trabajadores de oficina o planta de producción, o trabajadores a tiempo completo o tiempo parcial (Friedman y Miles, 2006). 
TABLA 2

CRITERIOS BÁSICOS DE CLASIFICACIÓN DE GRUPOS DE INTERÉS

\begin{tabular}{|c|c|c|c|}
\hline Autores & Criterios & Característica principal & Ejemplos \\
\hline \multirow[b]{2}{*}{ Freeman (1984) } & Internos & $\begin{array}{l}\text { Responsables de los cambios internos en } \\
\text { la compañía. La relación con estos grupos } \\
\text { es asequible. }\end{array}$ & $\begin{array}{l}\text { Trabajadores (empleados y directivos), } \\
\text { accionistas, proveedores y clientes. }\end{array}$ \\
\hline & Externos & $\begin{array}{l}\text { Responsables de los cambios externos a la } \\
\text { compañía. Influyen en los cambios internos } \\
\text { en la compañía. La relación con estos } \\
\text { grupos es más impredecible. }\end{array}$ & $\begin{array}{l}\text { Resto de grupos, como Gobiernos, } \\
\text { competidores, grupos ecologistas, } \\
\text { medios de comunicación, ciudadanos y } \\
\text { comunidades. }\end{array}$ \\
\hline \multirow{2}{*}{$\begin{array}{l}\text { Clarkson (1994), } \\
\text { en Mitchell et al. } \\
\text { (1997) }\end{array}$} & Voluntarios & $\begin{array}{l}\text { Soportan alguna forma de riesgo como } \\
\text { resultado de haber invertido algún tipo de } \\
\text { capital, humano o financiero, algo de valor, } \\
\text { en una empresa. }\end{array}$ & $\begin{array}{l}\text { Accionistas, entidades financieras, em- } \\
\text { pleados, proveedores, clientes. }\end{array}$ \\
\hline & Involuntarios & $\begin{array}{l}\text { Están expuestos a riesgos como resultado } \\
\text { de las actividades de una empresa. }\end{array}$ & Comunidades en general. \\
\hline \multirow{2}{*}{ Clarkson (1995) } & Primarios & $\begin{array}{l}\text { Necesarios para la continuidad de la } \\
\text { empresa. }\end{array}$ & $\begin{array}{l}\text { Inversores, empleados, clientes, provee- } \\
\text { dores, Gobiernos y comunidades. }\end{array}$ \\
\hline & Secundarios & $\begin{array}{l}\text { Relacionados con la empresa, pero no } \\
\text { esenciales para su supervivencia. }\end{array}$ & $\begin{array}{l}\text { Medios de comunicación, nichos de clien- } \\
\text { tela no atendida. }\end{array}$ \\
\hline \multirow{2}{*}{$\begin{array}{l}\text { Mitchell et al. } \\
\text { (1997) }\end{array}$} & Demandantes & $\begin{array}{l}\text { Tienen demandas morales, legales o presu- } \\
\text { mibles sobre la empresa. Tienen legitimidad. }\end{array}$ & \multirow{2}{*}{$\begin{array}{l}\text { Los ejemplos dependen de escenarios y } \\
\text { casuísticas específicas. }\end{array}$} \\
\hline & Influyentes & $\begin{array}{l}\text { Tienen la capacidad para influir en el } \\
\text { comportamiento, dirección, procesos y } \\
\text { resultados de la empresa. Tienen poder. }\end{array}$ & \\
\hline \multirow{4}{*}{$\begin{array}{l}\text { Mitchell et al. } \\
\text { (1997) }\end{array}$} & Definitivos & $\begin{array}{l}\text { Predominancia indiscutible y gran impor- } \\
\text { tancia para la empresa, por su poder, } \\
\text { legitimidad y urgencia. }\end{array}$ & \multirow{4}{*}{$\begin{array}{l}\text { Los ejemplos dependen de escenarios y } \\
\text { casuísticas específicas. }\end{array}$} \\
\hline & Expectantes & $\begin{array}{l}\text { Tendencia a permanecer activos frente a la } \\
\text { empresa. Combinan dos de los tres atribu- } \\
\text { tos anteriores. }\end{array}$ & \\
\hline & Latentes & $\begin{array}{l}\text { Solo poseen uno de los atributos anterio- } \\
\text { res, pero son susceptibles de incorporar } \\
\text { alguno más y volverse activos. }\end{array}$ & \\
\hline & Inactivos & $\begin{array}{l}\text { No considerados como grupos de interés } \\
\text { por parte de los directivos de la empresa. }\end{array}$ & \\
\hline \multirow{4}{*}{$\begin{array}{l}\text { Henriques y } \\
\text { Sadorsky (1999) }\end{array}$} & Regulatorios & $\begin{array}{l}\text { Entidades gubernamentales o con influen- } \\
\text { cia para generar normativa o regulaciones }\end{array}$ & $\begin{array}{l}\text { Gobiernos, asociaciones de comercio, re- } \\
\text { des de trabajo, competidores influyentes. }\end{array}$ \\
\hline & Organizaciones & $\begin{array}{l}\text { Relacionados directamente con la organiza- } \\
\text { ción y tienen alto poder de impacto en ella. }\end{array}$ & $\begin{array}{l}\text { Clientes, proveedores, empleados y } \\
\text { accionistas. }\end{array}$ \\
\hline & Comunidad & $\begin{array}{l}\text { Pueden movilizar la opinión pública a favor } \\
\text { o en contra de la organización. }\end{array}$ & $\begin{array}{l}\text { Comunidades, organizaciones ecologis- } \\
\text { tas, lobbies. }\end{array}$ \\
\hline & $\begin{array}{l}\text { Medios de } \\
\text { comunicación }\end{array}$ & $\begin{array}{l}\text { Pueden influir en la percepción que se tiene } \\
\text { sobre una compañía. }\end{array}$ & Medios de comunicación. \\
\hline
\end{tabular}

\section{Influencia de los grupos de interés en la actuación empresarial}

Podría resultar tentador para la empresa centrar su estrategia competitiva en iniciativas que persigan únicamente la creación de valor económico. Sin embargo, los actuales postulados en materia de competitividad y sostenibilidad de la empresa en el largo plazo apuntan hacia enfoques según los cuales la empresa estaría en la necesidad de crear valor social, además de económico, a través de los principios representados en el concepto de RSC (Porter y Kramer, 2006). Estos $D$ 
postulados están en consonancia con la llamada Triple Línea Base (Elkington, 1997), que afirma que las empresas, para ser sostenibles en el largo plazo, deben mostrar una actuación socialmente responsable que equilibre resultados positivos (creando valor), tanto económicos como sociales y medioambientales. En este caso se puede entender que, conceptualmente, una actitud socialmente responsable (aquella propia de un agente social responsable) incluye un esfuerzo por respetar y preservar el medioambiente (Bansal y Roth, 2000; Aguilera et al., 2007; Post et al., 2011).

La manera en que se define grupo de interés condiciona el enfoque con que se plantean otros conceptos relacionados con éste, destacando especialmente los principios de RSC que pueda adoptar la empresa (Kaler, 2003). Mientras que, según una definición ajustada de grupos de interés, la empresa debe atender las exigencias de los grupos de interés para poder operar, y esto implicaría atender cuestiones tanto prácticas como éticas, la definición amplia estaría más relacionada con la actuación ética de la empresa en materia de responsabilidad social, actuación que no sería de estricto obligado cumplimiento para poder continuar operando y existir, al menos en el corto plazo. Así, Mitchell et al. (1997), en referencia a la definición amplia de grupos de interés de Freeman, destacan que cuando no existen relaciones, transacciones o contratos entre grupos y empresa (algo que sí existe al considerar la definición ajustada), la empresa no percibe obligación de reciprocidad en su atención a dichos grupos. Además, resaltan que los atributos de los grupos de interés varían en el tiempo (comportamiento dinámico) y son de carácter social (no una realidad objetiva), y que la actividad de los grupos de interés no tiene por qué ser deliberada o consciente.
Utilizar, en cambio, un enfoque ajustado de grupos de interés en los planteamientos sociales de la empresa puede suponer que las empresas no identifiquen responsabilidades hacia la sociedad en general, sino específicamente hacia los grupos de interés que la representan (Clarkson, 1995; Donaldson y Preston, 1995; Maignan et al., 1999).

Los grupos de interés adoptan y desempeñan diferentes roles y tipos de actividades, intentando mediante ello influir en o presionar a las empresas para que adquieran compromisos y persigan comportamientos socialmente responsables (Aguinis y Glavas, 2012; Lee et al., 2013). Tres son los tipos de motivaciones para presionar a las empresas para que adopten compromisos sociales (y medioambientales): instrumental (por interés propio, por ejemplo, de ganar control), relacional (basado en las relaciones entre los miembros del grupo; por ejemplo, por cuestiones de identidad y pertenencia a colectivos) y moral (basada en los estándares de ética y principios morales, como, por ejemplo, el sentido de la justicia) (Aguilera et al., 2007). A su vez, estos grupos, pueden evaluar la actuación de las empresas de manera diferente, dependiendo no solo de su propio interés, sino también de su diferente ideología, o la forma de entender y aceptar los principios de RSC y su relación con los resultados de la empresa (Wood, 1991).

Wood y Jones (1995) identifican cuatro roles que pueden desempeñar los grupos de interés respecto a la empresa: (1) son fuente de expectativas sobre lo que se considera una actuación deseable e indeseable por parte de la empresa; (2) experimentan los efectos del comportamiento de la empresa: sus actuaciones y resultados; (3) evalúan el grado en que la empresa cumple con las expectativas planteadas y cómo los grupos y organizaciones se ven $\triangleright$ 
afectadas en su entorno; y por último, en consecuencia, (4) actúan basándose en los puntos anteriores. Esta situación se refuerza en cuanto a que la sociedad valora la actuación de las empresas y sus motivaciones a través de los grupos de interés en el contexto del sistema de valores de dichos grupos (Szwajkowski y Figlewicz, 1997).

Cuando las empresas adoptan un posicionamiento alineado con las exigencias de los grupos de interés, ganan legitimidad de actuación y supervivencia en el mercado. En el extremo contrario, los grupos de interés pueden someter a boicot (e incluso explotación) a las empresas, adoptando el papel de grupo activista. Los grupos de interés origen de estas iniciativas pueden ser de todo tipo, incluyendo directivos o accionistas que deseen una diferente redistribución de beneficios económicos. En entornos competitivos esa presión puede ser más perjudicial, ya que sus efectos destacan en mayor medida en el mercado, produciendo peores resultados en la empresa. Aunque un comportamiento ético de la empresa no la libra de posibles actos de boicot, las prácticas activas de RSC ayudan a las empresas a minimizar la posibilidad de ser objeto de ataque por parte de dichos activistas (Baron, 2001).

\section{Conclusiones}

El modelo clásico propuesto por la teoría de las organizaciones se queda obsoleto desde que se observa que las empresas están afectadas por la actuación de un gran número de grupos de interés que proceden del entorno empresarial. Al plantearse la necesidad de diseñar y establecer relaciones bidireccionales con ellos surge la teoría de los grupos de interés (Freeman, 1984).
La actividad de las empresas y su propuesta de valor afecta a los grupos de interés, tanto internos como externos a la organización, produciendo un impacto en su entorno social. Esto fuerza a dichos grupos a plantear una serie de peticiones o exigencias a las empresas, obligando a estas últimas a mostrar su compromiso respondiendo a las demandas planteadas mediante una propuesta de valor que las contemple. Así, las empresas consiguen legitimidad de actuación y supervivencia en el largo plazo (Brammer et al., 2007). Existen diferentes tipos de demandas hacia la empresa en el ecosistema que conforman los grupos de interés. Para cubrir todas ellas, la propuesta de valor debe contemplar las dimensiones económica, social y medioambiental.

La relación entre la actividad empresarial y el impacto en los grupos de interés, considerados pieza clave de su entorno social, determina el vínculo existente entre los conceptos de grupo de interés y RSC. El compromiso de las compañías con sus grupos de interés resulta visible y evaluable a través de las prácticas que, más allá de sus principios y planteamientos de RSC, finalmente desarrollen (Porter y Kramer, 2006). Las empresas muestran un mayor compromiso social cuanto mayor es el espectro de grupos de interés considerados y menor el grado de dependencia de la empresa respecto a estos grupos para su supervivencia y, por lo tanto, más reducida es su actuación por propio interés. En este último caso se dice que el posicionamiento social tiende a ser de tipo normativo o ético.

Una vez comprendido el concepto de grupo de interés, su importancia para las empresas, y la relación entre grupos de interés y RSC, es el turno de las empresas para diseñar un plan de gestión y diálogo con sus grupos de interés de acuerdo a su entorno de negocio. $\triangleright$ 
Los grupos de interés participan en la actividad de la empresa dependiendo de la medida en que ésta atiende a sus expectativas (Brammer y Pavelin, 2006). Es importante destacar que el nivel de exigencia para que las empresas actúen de un modo socialmente responsable va en aumento (Yim y Fock, 2013), y éstas deben ganarse la confianza y la legitimidad de los grupos de interés del entorno para operar (Brammer et al., 2007). Asumir una respuesta (y una consiguiente asignación de recursos) a todos los posibles grupos de interés existentes en el contexto de la empresa afectaría de manera severa a su capacidad competitiva, por lo que las compañías deben identificar y priorizar aquellos grupos de mayor relevancia (Hussain, 1999; Maak, 2007) o con mayor poder (Mitchell et al., 1997). Las fases por las que debería pasar una correcta gestión de grupos de interés son: (1) definición para la empresa de lo que entiende por grupo de interés; (2) enumeración y clasificación de los mismos, incluyendo asignación de prioridades; (3) estudio de los aspectos de relevancia para los grupos de interés y la capacidad de la organización para satisfacerlos, relacionado con el llamado análisis de materialidad; (4) diseño del plan de interacción con los diferentes grupos de interés; y (5) implementación del plan. Las empresas deben profundizar en estos aspectos, de gran importancia para aquellas que deseen operar con legitimidad, realizando un adecuado aporte de valor económico, social y medioambiental a su entorno.

\section{Bibliografía}

[1] AGUILERA, R.V.; RUPP, D.E.; WILLIAMS, C.A. y GANAPATHI, J. (2007). «Putting the S Back in Corporate Social Responsibility: A Multilevel Theory of Social Change in Organizations».
The Academy of Management Review, vol. 32, n. -3 , pp. 836-863. Academy of Management.

[2] AGUINIS, H. y GLAVAS, A. (2012). «What We Know and Don't Know About Corporate Social Responsibility: A Review and Research Agenda». Journal of Management, vol. 38, n.․ 4, pp. 932-968.

[3] AULD, G.; BERNSTEIN, S. y CASHORE, B. (2008). «The new corporate social responsibility». Annual Review of Environment and Resources, n. 3, pp. 413-435.

[4] BANSAL, P. y ROTH, K. (2000). «Why companies go green: A model of ecological responsiveness". Academy of Management Journal, vol. 43, n. ${ }^{\circ} 4$, pp. 717-736.

[5] BARON, D.P. (2001). «Private politics, corporate social responsibility, and integrated strategy". Journal of Economics \& Management Strategy, vol. 10, n. 1, pp. 7-45.

[6] BRAMMER, S.J.; MILLINGTON, A. y RAYTON, B. (2007). «The contribution of corporate social responsibility to organizational commitment». The International Journal of Human Resource Management, vol. 18, n.․10, p. 1701.

[7] BRAMMER, S.J. y PAVELIN, S. (2006). "Corporate Reputation and Social Performance: The Importance of Fit». The Journal of Management Studies, vol. 43, n.ํ 3, pp. 435455.

[8] CHRISTMANN, P. y TAYLOR, G. (2006). «Firm self-regulation through international certifiable standards: determinants of symbolic versus substantive implementation». Journal of International Business Studies, vol. 37, n. 6 , pp. 863-878.

[9] CLARKSON, M. (1994). «A risk based model of stakeholder theory". Proceedings of the second Toronto conference on stakeholder theory, pp. 18-19. Centre for Corporate Social Performance. University of Toronto.

[10] CLARKSON, M.B.E. (1995). «A stakeholder framework for analyzing and evaluating corporate social performance». The Academy of Management Review, vol. 20, n.․ 1, pp. 92-117. Academy of Management.

[11] DAVID, P.; BLOOM, M. y HILLMAN, A.J. (2007). «Investor activism, managerial responsiveness, and corporate social performance». Strategic Management Journal, vol. 28, n. 1 , pp. $91-100$. 
[12] DAVIDSON, W.N. y WORRELL, D.L. (1988). «The Impact of Announcements of Corporate Illegalities on Shareholder Returns». Academy of Management Journal, vol. 31, n.․ 1, p. 195.

[13] DONALDSON, T. y PRESTON, L.E. (1995). «The Stakeholder Theory of the Corporation: Concepts, Evidence, and Implications». The Academy of Management Review, vol. 20, n. -1, p. 65. Academy of Management.

[14] ELKINGTON, J. (1997). Cannibals with forks. Oxford, UK: Capstone.

[15] FREEMAN, R.E.(1984).Strategic Management: A Stakeholder Approach. Boston, MA: Pittman.

[16] FREEMAN, R.E. y REED, D.L. (1983). "Stockholders and Stakeholders: A New Perspective on Corporate Governance». California management review, vol. 25, n.․ 3, pp. 88.

[17] FRIEDMAN, A.L. y MILES, S. (2006). Stakeholders: Theory and practice. Oxford University Press on Demand.

[18] FRIEDMAN, B.A. (2009). «Human Resource Management Role Implications for Corporate Reputation». Corporate Reputation Review, vol. 12, n.ㅇ 3, pp. 229-244.

[19] GARRIGA, E. y MELÉ, D. (2004). «Corporate Social Responsibility Theories: Mapping the Territory». Journal of Business Ethics, vol. 53, n.. 1, pp. 51-71.

[20] GONZÁLEZ-MASIP, J. (2017). Efectos de las prácticas de responsabilidad social y medioambiental corporativas en la atracción y retención de talento. Madrid: Universidad Complutense de Madrid.

[21] GREENING, D.W. y GRAY, B. (1994). «Testing a model of organizational response to social and political issues". Academy of Management Journal, vol. 37, n. ${ }^{-}$3, p. 467.

[22] GREENWOOD, M. (2007). «Stakeholder Engagement: Beyond the Myth of Corporate Responsibility». Journal of Business Ethics, vol. 74, n.․ 4, pp. 315-327.

[23] HANSEN, S.D.; DUNFORD, B.B.; BOSS, A.D.; BOSS, R.W. y ANGERMEIER, I. (2011). «Corporate Social Responsibility and the Benefits of Employee Trust: A CrossDisciplinary Perspective». Journal of Business Ethics, vol. 102, n.ำ 1, pp. 29-45.

[24] HENRIQUES, I. y SADORSKY, P. (1999). «The relationship between environmental commitment and managerial perceptions of stakeholder importance». Academy of Management Journal, vol. 42, n. 1, pp. 87-99.

[25] HOPKINS, M. (2003). The Planetary Bargain: CSR Matters. London: Earthscan.

[26] HUSSAIN, S.S. (1999). «The ethics of 'going green': the corporate social responsibility debate". Business Strategy and the Environment, vol. 8, n. .4 , pp. 203-210.

[27] JONES, T.M.yWICKS, A.C.(1999). Convergent stakeholder theory. Academy of management review, vol. 24, n.ㅇ 2, pp. 206-221.

[28] KALER, J. (2003). «Differentiating stakeholder theories». Journal of Business Ethics, vol. 46, n. -1 , pp. 71-83.

[29] LEE, E.M.; PARK, S. y LEE, H.J. (2013). «Employee perception of CSR activities: Its antecedents and consequences». Journal of Business Research, vol. 66, n.. 10, p. 1716.

[30] MAAK, T. (2007). «Responsible Leadership, Stakeholder Engagement, and the Emergence of Social Capital». Journal of Business Ethics, vol. 74, n.․ 4, pp. 329-343.

[31] MAIGNAN, I.; FERRELL, O.C. y HULT, G.T.M. (1999). "Corporate citizenship: Cultural antecedents and business benefits". Journal of the Academy of Marketing Science, vol. 27, n.․ 4, pp. 455-469.

[32] MARQUIS, C.; GLYNN, M.A. y DAVIS, G.F. (2007). «Community isomorphism and corporate social action». The Academy of Management Review, vol. 32, n. 3, pp. 925-945.

[33] MITCHELL, R.K.; AGLE, B.R. y WOOD, D.J. (1997). «Toward a theory of stakeholder identification and salience: Defining the principle of who and what really counts». Academy of Management.The Academy of Management Review, vol. 22, n. .9 , pp. 853-886.

[34] NEVILLE, B.A.;BELL, S.J.y MENGÜÇ, B.(2005). «Corporate reputation, stakeholders and the social performance-financial performance relationship». European Journal of Marketing, vol. 39, n.․ (9/10), pp. 1184-1198,1216,1219-1220.

[35] OSTERWALDER, A. y PIGNEUR, Y. (2010). Business model generation: a handbook for visionaries, game changers, and challengers. John Wiley \& Sons.

[36] PHILLIPS, R. (1999). "On stakeholder delimitation». Business and Society, vol. 38, n. 1, pp. 32-34. 
[37] PORTER, M.E. y KRAMER, M.R. (2006). «Strategy \& Society: The Link between Competitive Advantage and Corporate Social Responsibility». Harvard Business Review, vol. 84, n. -12 , pp. 78-92.

[38] POST, C.; RAHMAN, N. y RUBOW, E. (2011). «Green Governance: Boards of Directors' Composition and Environmental Corporate Social Responsibility". Business and Society, vol. 50, n.․ 1, pp. 189.

[39] RHENMAN, E. (1968). Industrial Democracy and Industrial Management. London: Tavistock Publications.

[40] SEN, S. y BHATTACHARYA, C.B. (2001). «Does doing good always lead to doing better? Consumer reactions to corporate social responsibility». Journal of Marketing Research, vol. 38, n.․ 2, pp. 225-243.

[41] SER (2000). Corporate Social Responsibility: part of the core business. Assen, Netherlands: Sociaal Economische Raad/Social Economic Council. Disponible en: https://www.ser.nl/en/ publications/publications/2000.aspx

[42] STARIK, M. (1995). "Should trees have managerial standing? Toward stakeholder status for non-human nature». Journal of Business Ethics, vol. 14, n.ㅇ 3, pp. 207-217.

[43] STARIK, M. y RANDS, G.P. (1995). «Weaving an integrated web: Multilevel and multisystem perspectives of ecologically sustainable organizations». Academy of Management Review, vol. 20, n.․ 4, pp. 908-935.

[44] SZWAJKOWSKI, E. y FIGLEWICZ, R.E. (1997). "Of Babies and Bathwater. An Extension of the Business \& Society Research Forum on the Fortune Reputation Database». Business \& Society, vol. 36, n.․ 4, pp. 362-386.
[45] VAN MARREWIJK, M. (2003). «Concepts and definitions of CSR and corporate sustainability: Between agency and communion". Journal of Business Ethics, vol. 44, n. (2/3), pp. 95-105.

[46] WADDOCK, S.; BODWELL, C. y GRAVES, S.B. (2002). «Responsibility: The new business imperative». The Academy of Management Executive, vol. 16, n.을, pp. 132-148.

[47] WATTS, P. y HOLME, L. (2000). Corporate Social Responsibility: Making Good Business Sense. Geneva: World Business Council for Sustainable Development.

[48] WEAVER, G.R.; TREVINO, L.K. y COCHRAN, P.L. (1999). «Corporate ethics programs as control systems: Influences of executive commitment and environmental factors". Academy of Management Journal, vol. 42, n. 1, pp. 41-57.

[49] WOOD, D.J. (1991). «Corporate Social Performance Revisited». Academy of Management. The Academy of Management Review, vol. 16, n.․․ 4, pp. 691.

[50] WOOD, D.J. (2010). «Measuring Corporate Social Performance: A Review». International Journal of Management Reviews, vol. 12, n. -1 , pp. 50-84.

[51] WOOD, D.J. y JONES, R.E. (1995). «Stakeholder mismatching: A theoretical problem in empirical research on corporate social performance». The International Journal of Organizational Analysis, vol. 3, n. 3, pp. 229-267.

[52] YIM, F. y FOCK, H. (2013). «Social Responsibility Climate as a Double-Edged Sword: How Employee-Perceived Social Responsibility Climate Shapes the Meaning of Their Voluntary Work?». Journal of Business Ethics, vol. 114, n. - 4, pp. 665-674. 

BOLETÍN ECONÓMICO DE INFORMACIÓN COMERCIAL ESPAÑOLA (BICE) ISSN 0214-8307

\section{SUSCRIPCIÓN ANUAL}

\begin{tabular}{|c|c|c|c|}
\hline \multicolumn{2}{|c|}{ BOLETÍN ECONÓMICO DE INFORMACIÓN COMERCIAL ESPAÑOLA (12 NÚMEROS) } \\
\hline & $\begin{array}{c}\text { ESPAÑA } \\
\mathbf{1} \text { año }\end{array}$ & $\begin{array}{c}\text { UNIÓN EUROPEA } \\
\mathbf{1} \text { año }\end{array}$ & $\begin{array}{c}\text { RESTO DEL } \\
\text { MUNDO } \\
1 \text { año }\end{array}$ \\
\hline SUSCRIPCIÓN & $65,00 €$ & $85,00 €$ & $85,00 €$ \\
\hline $\begin{array}{c}\text { Gastos de envío } \\
\text { España }\end{array}$ & $5,76 €$ & $24,36 €$ & $30,00 €$ \\
\hline $\begin{array}{c}\text { Más 4\% de IVA. } \\
\text { Excepto Canarias, Ceuta y Melilla }\end{array}$ & $2,83 €$ & & $115,00 €$ \\
\hline TOTAL & $\mathbf{7 3 , 5 9 €}$ & $\mathbf{1 0 9 , 3 6} €$ & \\
\hline & & & \\
\hline
\end{tabular}

\section{EJEMPLARES SUELTOS}

\begin{tabular}{|c|c|c|c|}
\hline BOLETÍN ECONÓMICO & DE INFORMACIÓN & COMERCIAL ESPAÑOL & \\
\hline & $\begin{array}{l}\text { ESPAÑA } \\
1 \text { ejemplar }\end{array}$ & $\begin{array}{l}\text { UNIÓN EUROPEA } \\
1 \text { ejemplar }\end{array}$ & $\begin{array}{l}\text { RESTO DEL } \\
\text { MUNDO } \\
1 \text { ejemplar }\end{array}$ \\
\hline NÚMERO SUELTO & $7,00 €$ & $9,00 €$ & $9,00 €$ \\
\hline $\begin{array}{l}\text { Gastos de envío } \\
\text { España }\end{array}$ & $0,48 €$ & $2,03 €$ & $2,50 €$ \\
\hline $\begin{array}{l}\text { Más } 4 \% \text { de IVA. } \\
\text { Excepto Canarias, Ceuta y Melilla }\end{array}$ & $0,30 €$ & & \\
\hline TOTAL & $7,78 €$ & $11,03 €$ & $11,50 €$ \\
\hline \multirow[t]{2}{*}{ BOLETÍN ECONÓMICO } & DE INFORMACIÓN & COMERCIAL ESPAÑOL & \\
\hline & $\begin{array}{l}\text { ESPAÑA } \\
1 \text { ejemplar }\end{array}$ & $\begin{array}{l}\text { UNIÓN EUROPEA } \\
1 \text { ejemplar }\end{array}$ & $\begin{array}{c}\text { RESTO DEL } \\
\text { MUNDO } \\
1 \text { ejemplar }\end{array}$ \\
\hline $\begin{array}{l}\text { NÚMERO SUELTO } \\
\text { EXTRAORDINARIO }\end{array}$ & $12,00 €$ & $15,00 €$ & $15,00 €$ \\
\hline Gastos de envío España & $0,48 €$ & $2,03 €$ & $2,50 €$ \\
\hline $\begin{array}{c}\text { Más 4\% de IVA. } \\
\text { Excepto Canarias, Ceuta y Melilla }\end{array}$ & $0,50 €$ & & \\
\hline TOTAL & $12,98 €$ & $17,03 €$ & $17,50 €$ \\
\hline
\end{tabular}

\section{DATOS}

Nombre y apellidos

Empresa

Domicilio

D.P.

N.I.F.

Teléf.

Email
DATOS DEL EDITOR:

NIF:S2800568D

Transferencia a la cuenta de ingresos por venta de publicaciones del Ministerio de Economía y Competitividad.

IBERCAJA. Calle Alcalá 29. 28014 MADRID (ESPAÑA) CÓDIGO CUENTA CLIENTE: 2085-9252-07-0330598330 CÓDIGO BIC DE IBERCAJA: CAZRES2Z

IBAN: ES47 2085-9252-07-0330598330

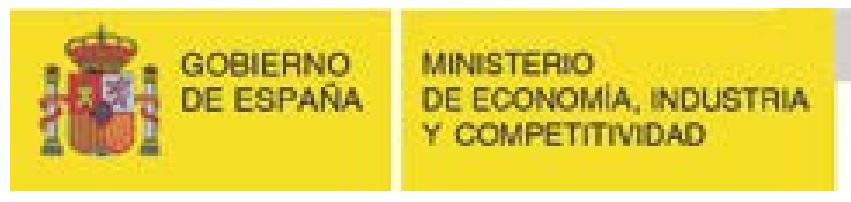

\section{SUESECAETARIA}

SECRETARIA GENERAL TECNICA

DUECIRECCION GENTRM OS

ESTuONOS, BNFOSACXCN Y PUSUCACONES

Suscripciones y ventas por correspondencia:

Paseo de la Castellana, 162, 9ª Planta, 28046 Madrid. Teléfono 916037993 / 97

Suscripciones a través de la página web del Ministerio de Economía, Industria y Competitividad distribucionpublicaciones@mineco.es 


\section{NORMAS DE ESTILO DE PUBLICACIÓN}

La persona o personas interesadas en presentar un artículo para su publicación en el Boletín de Información Comercial Española (BICE) deberán enviar el artículo en formato Microsoft Word a la dirección de correo electrónico revistasice.sscc@comercio.mineco.es

El documento debe cumplir las siguientes características:

1. Ser material original no publicado ni presentado en otro medio de difusión.

2. La extensión total del trabajo (incluyendo cuadros, gráficos, tablas, notas, etcétera) no debe ser inferior a 15 páginas ni superior a 20. La fuente será Times New Roman, tamaño 12 y espaciado doble. Estar paginado en la parte inferior derecha de cada página.

3. En la primera página se hará constar el título del artículo que deberá ser breve, claro, preciso e informativo y la fecha de conclusión del mismo. Nombre y dos apellidos del autor o autores, filiación institucional, dirección, teléfono y correo electrónico de cada uno de ellos, así como la forma en que desean que sus datos aparezcan.

4. En la segunda página del texto se incluirá:

- El título.

- Un resumen del trabajo con una extensión máxima de 10 líneas con la siguiente estructura: objetivo, método y principal resultado o conclusión.

- De 2 a 6 palabras clave que no sean coincidentes con el título.

- De 1 a 5 códigos de materias del Journal of Economic Literature (clasificación JEL) para lo cual pueden acceder a la siguiente dirección electrónica:

https://www.aeaweb.org/jel/guide/jel.php

5. En las siguientes páginas se incluirán el texto, la información gráfica y la bibliografía con la siguiente organización.

- Se incluirá, por este orden, introducción, desarrollo, conclusiones y bibliografía y anexos si los hubiera. Los apartados y subapartados se numerarán en arábigos respondiendo a una sucesión continuada utilizando un punto para separar los niveles de división, según el siguiente modelo:

\section{Título del apartado}

\subsection{Título del apartado}

\subsubsection{Título del apartado}

\section{Título del apartado}

- Las notas de pie de página irán integradas en el texto y su contenido debe estar al final de su misma página en tamaño 10 y espacio sencillo.

- En un archivo Excel independiente se incluirá la representación gráfica (cuadros, gráficos, diagramas, figuras, etc.), que debe llevar título, estar numerada y referenciada en el texto.

En la parte inferior se incluirá la fuente de información y/o notas aclaratorias.

- Las citas de libros y artículos en el texto, se indicarán entre paréntesis con el apellido del autor y el año. Ej.: (Martínez, 1991).

- Las referencias a siglas deben ir acompañadas, en la primera ocasión en que se citen, de su significado completo.

- La bibliografía se ordenará alfabéticamente siguiendo las normas de la American Psychological Association (Harvard-APA): http://cibem.org/paginas/img/apa6.pdf

Libros

APELLIDOS, A.A. (año de publicación). Título del libro (edición) (volumen). Ciudad: Editorial.

Artículo en revista científica

APELLIDOS, A.A. (año de publicación). «Título del artículo». Título de la revista, volumen (número), números de páginas.

\section{Documento en línea}

APELLIDOS, A.A. u ORGANISMO (año, mes de publicación). Título, [en línea]. Ciudad: Editorial. Disponible en:

http://cenamb.rect.ucv.ve/siamaz/dicciona/canaima/canaima2.htm [Recuperado: 2000, 3 de junio]. 